\title{
Entre doutes et engagements : un arrêt sur image à partir de l'histoire des femmes
}

\author{
Myriam Cottias, Cécile Dauphin, Arlette Farge, Nancy L. Green, Danielle \\ Haase-Dubosc, Danièle Poublan et Yannick Ripa
}

\section{(2) OpenEdition}

Édition électronique

URL : https://journals.openedition.org/clio/1383

DOI : $10.4000 /$ clio. 1383

ISSN : 1777-5299

Éditeur

Belin

Édition imprimée

Date de publication : 1 novembre 2004

Pagination : 231-260

ISBN : 2-85816-755-9

ISSN : $1252-7017$

Référence électronique

Myriam Cottias, Cécile Dauphin, Arlette Farge, Nancy L. Green, Danielle Haase-Dubosc, Danièle Poublan et Yannick Ripa, «Entre doutes et engagements : un arrêt sur image à partir de l'histoire des femmes », Clio. Histoire, femmes et sociétés [En ligne], 20 | 2004, mis en ligne le 01 janvier 2007, consulté le 22 avril 2022. URL : http://journals.openedition.org/clio/1383 ; DOI : https://doi.org/ 10.4000/clio.1383

Ce document a été généré automatiquement le 22 avril 2022.

Tous droits réservés 


\title{
Entre doutes et engagements : un arrêt sur image à partir de l'histoire des femmes
}

\author{
Myriam Cottias, Cécile Dauphin, Arlette Farge, Nancy L. Green, Danielle \\ Haase-Dubosc, Danièle Poublan et Yannick Ripa
}

1 Quelque chose d'un désenchantement, d'une opacité, ou peut-être d'une inquiétude gagne l'ensemble de la recherche: les événements s'accélèrent, les générations se renouvellent, les peurs et les incertitudes sont de plus en plus marquées, et dans ce contexte difficile, tout ce qui ressemble à un engagement, à une conviction, fussent-ils subtils, est soupçonné. La résignation ou une certaine insignifiance forme le contenu de travaux historiques qui ne trouvent pas de véritable ampleur ou acuité depuis quelques années.

Le Groupe d'Histoire des Femmes ${ }^{1}$, comme chaque groupe de recherche, subit ce contexte historique de plein fouet et se retrouve confronté à la complexité grandissante du présent et à une distorsion entre le « réel » et l'insuffisance des outils d'analyse, par une rupture d'intelligibilité. L'histoire des femmes a certes donné ses preuves; il y eut de nombreux travaux: la parution en 1991 des cinq volumes de l'Histoire des Femmes ${ }^{2}$, des séminaires de recherche en université, des colloques, des livres marquants ${ }^{3}$, des événements et des rencontres. Qu'importe, quelque chose s'est gelé ou reste insatisfaisant: une absence par rapport aux enjeux actuels du domaine social et politique fait ressortir les préoccupations féministes françaises comme autant de thèmes peu adéquats face aux angoisses contemporaines (terrorisme, mondialisation, guerres, violence sur les femmes, chômage), aggravées par la disparition des grands thèmes porteurs de la sociologie (lutte des classes, domination, etc.). Face à cela, pourtant, des jeunes (Mix-Cité, Ni putes ni soumises) trouvent (ou cherchent et trouvent) d'autres voies, d'autres chemins, d'autres paroles, délaissant ce que furent les thèmes des combats des années 1970. En même temps, il faut le reconnaître, assez peu d'attention aux différences entre classes sociales, et l'idée de fait erronée selon laquelle la femme pourrait représenter une entité générique ont fait 
perdre beaucoup d'efficacité aux propos émis. La "sororité » d'antan s'est brisée en mille morceaux devant tant de conflits ouverts, tant de raccourcis idéologiques assumés et qui avaient peu d'efficacité pratique pour la vie de tous les jours, tant de différences accrues et non-dites entre milieux sociaux. Qui peut oser comparer les intellectuelles parisiennes en vue et médiatisées, et leur volonté de discréditer la critique féministe, à l'ouvrière décrite récemment dans un livre de Franck Magloire ${ }^{4}$.

Quant aux jeunes générations des classes moyennes, on ne s'en est sans doute pas assez souciées; elles prennent l'apparence de n'avoir rien à dire sur le sujet et ont, dit-on, intégré ou intériorisé ce qui fut autrefois le combat de leurs mères. Les filles ont pu s'approprier les acquis maternels mais ressentent aujourd'hui bien des défaillances de leurs discours. Sur les cultures amoureuses que les jeunes vivent actuellement, il y aurait beaucoup à dire, ou plutôt « ils » auraient beaucoup à dire. La démocratie et le libéralisme marchant la main dans la main, les jeunes vivent d'autres facilités, besoins, envies, résignations que leurs aînés. L'éphémère y est très présent, et les temporalités vécues bien différentes. Le désir marchand et les corps marchandises font la une des journaux, des médias, des images, cela devant les grands conflits de notre société (ghettos de banlieue, femmes voilées, exclus de plus en plus nombreux dormant dans nos rues). Bien des jeunes femmes mélangent dans une forte tension le désir d'indépendance et l'envie de reprendre quelques joies (ou facilités) du machisme ordinaire : dépendance, protection, virilité. Le SIDA fait peur aux corps masculins et féminins. Devant le brouillage des rôles, certains jeunes expriment de la souffrance, et pendant ce temps un vocabulaire manque au féminisme et surtout une appréhension subtile du monde des passions, des affects, et des constructions des altérités.

Alors il faut accepter de réfléchir et surtout d'inventer, puisque les repères nous manquent. Ce que nous voulons présenter ici a une forme tout à fait spécifique et inattendue, qui ne ressemble pas aux articles de la recherche la plus habituelle. En effet, à plusieurs, mais chacune selon l'axe de ses intérêts et de ses préoccupations intellectuelles, nous avons décidé de réfléchir sur quelques mots clés, notions ou concepts, qui ont construit une partie de la recherche en histoire des femmes, et de suivre leur trajectoire jusqu'à aujourd'hui, soit parce que ces mots n'ont plus aucune efficacité et qu'il faut comprendre pourquoi, soit parce que d'autres non employés dans les années du féminisme triomphant doivent actuellement être profondément pensés. Bien entendu, il n'y a rien d'exhaustif en cette liste de mots, de concepts ou notions; il s'agit d'une réflexion qui s'impose pour continuer nos recherches, discuter avec celles et ceux qui nous liront, reprendre le débat et surtout la force d'aller à la fois au plus loin de la créativité et au plus près de celles et ceux qui attendent un autre discours sur l'égalité des sexes.

5 Comment tracer un chemin qui tiendrait compte de l'idéal féministe dans la discipline historique et comment repenser même le mot féminisme ? Beaucoup reste à faire sur la masculinité comme objet d'histoire, sur le monde des passions, sur les formes du désir. Par ailleurs le concept de genre a ses limites: devenu stéréotypal, il n'est pas assez politique et largement atemporel. Le problème fondamental est sans doute de trouver des outils pour aborder ce qui échappe au langage, au verbe et ce qui s'inscrit dans les savoirs, les mémoires et les corps. Les émotions et les souffrances, les violences inhumaines fabriquent d'autres langages, peut-être même d'autres logiques. Néanmoins, l'urgence à présent est de ne plus séparer ce surgissement infini et inouï de 
douleurs et de larmes de l'histoire politique ou géopolitique, sensée le rationaliser et en faire une conséquence inévitable.

6 Pour cela, cette incursion dans quelque mots, notions ou concepts très utilisés il y a peu dans le discours de l'histoire des femmes, nous semble une mise au point rapide mais qui donne néanmoins un éclairage sur ce qui fut et doit, ou non, être dépassé. Il s'agit ici non d'une prospective, ni même d'un bilan, mais d'une posture réflexive qui se veut un moyen de travailler plus efficacement, en comprenant certaines inadéquations passées et en englobant à bras le corps le monde d'aujourd'hui. Qu'on n'y cherche pas un programme ou une élucidation mais un arrêt sur images, une critique du vocabulaire et des notions, un désir de politique et d'appréhension plus fine du réel. Cette posture intellectuelle a permis à chaque membre de ce groupe de choisir une notion, un concept ou un mot pour interroger son usage et ses effets dans les problématiques de la rencontre entre masculin et féminin. Ce qui donne à cet article une forme insolite et non académique: de courts textes se suivent sans transition, assumés par chacune des signataires. Leur scansion est fidèle au mouvement de nos interrogations entre engagement, écriture scientifique de l'histoire et inquiétudes.

Les métaphores de la sexualité et de la liberté

Depuis le $\mathrm{XIX}^{\mathrm{e}}$ siècle le vocabulaire de l'esclavage comme forme extrême de domination, a été repris par les pionnières du féminisme. Entre 1830 et 1848, un certain nombre d'ouvrages utilise, en effet, la rhétorique anti-esclavagiste pour dénoncer la situation des femmes et placer les hommes dans la catégorie des « maitres/colons » des Antilles. En 1832, Jeanne Deroin ${ }^{5}$, déclare que « l'esclavage de la femme est un privilège odieux fondé sur le droit du plus fort » tandis que Claire Demar écrit la même année un ouvrage intitulé Appel d'une femme au peuple pour l'affranchissement de la femme. Dans une période où le gouvernement de Louis-Philippe établissait des lois instaurant l'égalité civile pour la population libre de couleur, discriminée dans les Colonies françaises des Antilles à cause de sa couleur et de son appartenance sociale, et qu'en même temps, il ouvrait les débats sur l'abolition de l'esclavage, le journal intitulé L'apostolat des femmes changeait de titre pour porter celui de l'Affranchissement des femmes. Cette corrélation étroite a été encore plus marquée dans le monde anglo-saxon, plus ouvertement posée en termes politiques. Des sociétés féministes protestantes ont mêlées leurs revendications de liberté à celles des esclaves tel ce Plaidoyer d'une moitié de la race humaine, les femmes, contre les prétentions de l'autre moitié, les hommes, à les maintenir dans un esclavage politique et par conséquent civil et domestique ${ }^{6}$. Si l'émancipation des esclaves est promulguée en France, en 1848, le concept d'esclavage est demeuré cependant l'outil pour penser la domination sexuée. Dans ce qui pourrait paraître comme un déplacement ou une superposition des enjeux, des femmes françaises ont d'ailleurs investi la Société d'abolition de l'esclavage qui a continué à s'intéresser, après la France, à la Russie, à l'Empire Ottoman, au Brésil ou encore aux États-Unis. Au long des siècles, l'esclavage comme archétype de relations sociales iniques et arbitraires a continué à imprégner les analyses des relations de genre.

8 Cette affirmation doit cependant être précisée car ce moteur d'analyse a connu des phases de résurgence ou de disparition. Organisés en phases cycliques, ces moments révèlent les avatars de la pensée politique, de l'analyse sociale tout autant que les tensions entourant le genre. De la fin du XIXe siècle jusqu'aux années 1960, esclavage et genre ne sont plus strictement corrélés : d'une part, sous l'influence du marxisme qui transforme les catégories de pensée, et de l'autre, sous la pression du contexte 
familialiste et des conditions démographiques de l'après-guerre qui assimilent la femme à la mère. D'un côté, l'histoire de l'humanité est celle de la lutte des classes et non celle de la lutte des sexes, de l'autre, l'avenir de l'homme, c'est la femme ! C'est au moment des mouvements de décolonisation que les luttes de domination retrouvent leurs références antérieures, prises entre dominé-noir et dominée-blanche. Les discours sur la lutte politique contre la domination coloniale se retrouvent comme antérieurement simultanés et parallèles à la lutte contre le patriarcat comme rapport social de subordination du féminin au masculin. La métaphore de la « noirceur » teinte les discours. À côté d'une enclave psychanalytique où la sexualité féminine est décrite comme le "continent noir " par Freud, la "féminitude" est forgée à partir de la «négritude » par Simone de Beauvoir. Le combat politique dans les années 70, tout à la fois «s'essentialise » et se «racialise » de façon consentie. Le féminisme se pose alors, de la même façon que l'anti-colonialisme, comme une revendication et une prise en compte de l'altérité.

9 La divergence des deux idéologies a succédé à cet enchevêtrement rhétorique et symbolique sous la pression des acquis politiques et sociaux. La satisfaction partielle et/ou illusoire des revendications a marqué une césure nette. "Libération des peuples » et "libération des femmes" sont reconnues comme légitimes. En France, l'affichage d'une législation consacrée aux femmes (pilule, IVG, viol, discriminations sexuelles, divorce, égalité des salaires...) lénifie aux yeux de beaucoup les rapports entre les sexes. La nouvelle catégorie de "femme libérée » sur le plan de la sexualité est de plus en plus mise en avant concomitamment à la défaite des systèmes idéologiques. Dans le même mouvement, toutes les pistes menant au social sont aussi brouillées et on peut lire dans le journal Femmes en Mouvement que la princesse Soraya, en tant que femme, a la même expérience que l'ouvrière de chez Renault ! L'expérience sexuelle en tant qu'expérience de la domination masculine et comme facteur ontologique de cette catégorie, abrase toutes les différences sociales habituant les esprits à une assurance certaine d'une division bi-partite du Monde, femmes versus hommes. C'est précisément dans cet essentialisme que le mouvement féministe a rencontré des écueils.

Alors que le corps des femmes continuait et continue à être un enjeu politique ${ }^{7}$, l'activité sexuelle s'est progressivement constituée comme un domaine moral selon un des questionnements posés par Foucault dans l'Usage des plaisirs ${ }^{8}$. En effet, la mise en discours de la sexualité par les féministes que ce soit dans des ouvrages déjà anciens (Germaine Greer, Kate Millet, pour les États-Unis, Annie Leclerc, pour la France), ou dans des vulgates, a conduit à ce que, dans une logique égalitaire, le sexuel ne pouvait s'accomplir que dans la complicité et la tendresse. Cette posture « révolutionnaire » et nécessaire à l'époque, a cependant engendré une sorte de moralisme dont les effets se font sentir aujourd'hui. Dans les années 70, les discours sur la sexualité ont décliné des thèmes allant du lesbianisme comme forme de relation parfaite à l'assimilation du mariage hétérosexuel à la prostitution'. Colette Guillaumin forme dans ces années le terme de « sexage » à partir de celui d'« esclavage ». C'est ainsi que si le féminisme s'est ancré autour de la «liberté sexuelle ", c'est aussi autour de la question de la sexualité qu'il s'est fragmenté. En effet, les principales fractures se sont produites à partir du cadre idéologique formé autour de la sexualité, car une multitude de sentiments échappaient aux analyses des relations sociales et de genre, à de très rares exceptions et sur ce point il faut saluer le travail et les pressantes recommandations d'Arlette Farge. Auraient pu être étudiés le désir et la réciprocité du désir entre un homme et une femme ${ }^{10}$, la jouissance féminine dans sa relation à un homme, les fantasmes et le 
désir d'être dominé ou de dominer, le consentement à l'inégalité pour préserver l'image virile de leur partenaire ${ }^{11}$. Le corps des femmes n'est pas que politique; il est l'objet de plusieurs formes d'assignation. C'est précisément à travers (ou à cause) de ces indicibles que le vocabulaire et la problématique sur l'esclavage et les races sont réapparus depuis quelques années dans toutes les tendances du féminisme et, plus largement dans les discours sur la pornographie et sur la prostitution, mais avec un déplacement des altérités. Il ne s'agit plus, en effet, de dénoncer un rapport de domination forgé sur une analyse des relations déséquilibrées Nord-Sud, par exemple, mais de considérer la pornographie et la prostitution à partir de la notion de «choix individuel » devenant par une arithmétique non établie, « le choix des femmes ».

11 Illustrant ce propos, certaines femmes se déclarant féministes avancent ainsi l'argument que la pornographie est un facteur d'épanouissement personnel et d'égalité politique, économique ou sociale ${ }^{12}$ et que la prostitution est " travail du sexe ». À aucun moment cependant, elles ne mettent en doute épanouissement personnel et égalité sociale. Brigitte Lahaie, Tabatha Cash ou encore Ogivie, réalisatrice de films pornographiques et auteure de Pro-manifesto ${ }^{13}$ se déclarent d'ailleurs des féministes pro-sexe. Depuis quelques années, il y a eu certes une appropriation par les femmes d'une sexualité dont les formes étaient marquées jusqu'alors par le masculin. Virginie Despentes, dans son roman Baise-moi comme dans son film éponyme, a illustré la violence de deux femmes dans les relations sexuelles; Catherine Breillat a montré le jeu d'une femme autour de la soumission pour arriver au plaisir dans son film Romance $X$; Catherine Millet a fait minutieusement et élégamment dans $L a$ vie sexuelle de Catherine $M$. le catalogage de ses expériences sexuelles mais sans la sensualité ni l'imaginaire d'illustres précédents comme Histoire d'O de Pauline Réage. Progressivement, l'image prostitutionnelle en tant que pratique symbolique et sublimée -que ce soit dans les discours artistiques ou dans la mode-, est devenue la métaphore généralisée et englobante de la sexualité consentie grâce à la revendication du choix.

Si l'on peut bien admettre la polysémie du terme «féministe » et ses appropriations diverses et contradictoires (et peut-être serait-il intéressant de les mettre toutes en perspective), il est plus inquiétant de voir selon quelles lignes de fracture évoluent les notions d'«abolitionnisme » et son contraire qui ne s'appelle pas « esclavagisme » mais "pro-prostitution»! La ligne des oppositions pour appréhender les rapports de genre s'est en effet déplacée progressivement. Depuis les années 60 , on est passé du binôme (Noirs-Femmes) contre (Blancs Occidentaux-Hommes) à un resserrement autour de celui de (Femmes/Hommes) pour aboutir actuellement à une opposition construite autour de (Femmes étrangères/victimes) contre (Femmes françaises/ Hommes libres) ${ }^{14}$. En effet, en écartant de leurs propos les femmes soumises à la traite de l'Est ou de l'Ouest africain ${ }^{15}$, les féministes "pro-prostitution » situent la ligne du consentement par rapport à la nationalité. Borné par le «choix » et la «liberté » (dont il faudrait démontrer l'équivalence), un entre-nous nationaliste est ainsi construit, révélant outrageusement une idéologie bien déformée par rapport à celui des origines.

Dans une mise en abîme intéressante, la question de l'altérité évacuée par ces discours a cependant ressurgi au cœur même de la Nation, chez les oubliés des banlieues issus, pour la plupart, de l'histoire coloniale. Depuis quelques années, brutalement, les thèmes de "choix» et de "liberté " ont fait ressurgir les différences sociales et culturelles à travers les définitions que ces termes avaient. Les mouvements récents comme celui de « Mix-cité » ou de « Ni putes, ni soumises » sont apparus, en effet, dans 
la nécessité de compléter des discours dans lesquels ils ne pouvaient pas se reconnaître et aussi parce qu'il fallait affirmer une réalité toute autre. Bien que française, le choix pouvait se résumer pour une femme à « être voilée » ou à " être une pute »; et la liberté à rester chez soi ou à être brûlée. La revendication de la contraception pouvait encore y être d'actualité : le Livre blanc des femmes de quartier réclame «le droit à l'éducation sexuelle pour tous (information sur le corps, la contraception et les MST - maladies sexuellement transmissibles). Alors n'y a-t-il pas urgence? Pour répondre à cette question, d'une part, un des devoirs des sciences humaines par rapport au masculin et au féminin est de ré-interroger les impensés ou les indicibles de la sexualité en les historicisant, et de faire entrer la palette des sentiments et des affects, même perturbants, à l'intérieur des contextes sociaux et politiques ${ }^{16}$. De l'autre, il faut qu'un ensemble national multiforme repense à présent les différences sociales et culturelles qu'il organise. Il faut prendre en compte la différenciation des expériences sociales car placer la liberté du choix individuel au centre des organisations sociales fait la douteuse économie de la véritable analyse des rapports d'inégalité.

Le consentement

Le consentement est une notion qui ne pouvait pas faire partie du vocabulaire des études féministes pionnières. Qu'auraient-elles fait de ce concept alors que la réalité était justement de ne plus, de ne pas consentir, de s'opposer, de montrer à quel point le désaccord était fort? On peut affirmer, sans trop se tromper, que c'est autour du refus que se cristallise l'action militante et intellectuelle, et c'est, bien entendu, sur l'affirmation d'un non-consentement et la recherche d'un autre devenir que se créèrent les premiers travaux. S'ils étaient, comme on disait à l'époque, à la recherche d'un nouveau futur, il ne pouvait être question d'un consentement à ce qu'on appelait alors la condition féminine. Ceci d'ailleurs - il faut être franche et claire - n'était pas exprimé (ni même expliqué en ces termes), puisque l'argument principal des recherches était celui d'une condition féminine (ouvrière, domestique, vie sexuelle, maternité) qui tournait autour d'un axe (qu'on dit simple aujourd'hui), celui de la domination et de l'oppression. Comment donc "penser » au consentement : ce mot même n'avait aucun sens. Ce n'était pas (d'ailleurs, y pensait-on?) une donnée bonne à penser. Il faut absolument, pour comprendre ce que furent les études féminines-féministes, savoir accomplir un travail d'historien(ne) sur le contexte. 1970 est loin, 2004 est si proche, si différent. Personne sans doute - et qui le leur reprocherait - ne pouvait penser à cette notion du «consentir» alors que rébellion et remise en question du statu quo, de l'infinie domination étaient le mot d'ordre.

Depuis, les problématiques de l'histoire sociale comme celles de l'histoire culturelle ont renouvelé bien des questionnements dans la discipline historique. Par exemple, dans ce domaine si particulier et qui fut si débattu de la définition de la culture populaire par rapport à la culture savante, on en vint a réfléchir sur les interactions entre ces deux types de culture. L'ensemble des historiens des représentations et d'une nouvelle histoire culturelle s'opposèrent à certains de leurs aînés qui voyaient dans la culture populaire un système d'aliénation et un succédané d'une culture savante surplombante (notions et concepts qui, d'ailleurs, se ré-élaborent régulièrement).

Dans l'histoire des femmes, la notion clé de la domination et de l'oppression régissant l'ordre du masculin et du féminin ne fut que très tardivement remise en cause, ou plutôt infléchie. En effet, au cœur de cette hiérarchisation entre masculin et féminin 
pouvaient survenir (et c'est encore l'histoire culturelle qui permit ces timides démarches) des formes de pouvoir empruntées par les femmes, placées dans les interstices du pouvoir dominant masculin. Un livre dirigé par Danielle Haase-Dubosc et Eliane Viennot ${ }^{17}$ vint montrer quelques types de prises de pouvoir.

18 Auparavant, de brillantes études ethnologiques et ethnographiques (Françoise Zonabend, Yvonne Verdier ${ }^{18}$ ) avaient essayé de saisir les complémentarités entre rôle masculin et rôle féminin plutôt que les oppositions ou les dominations. Ces études furent réalisées sur un domaine rural (Minot dans le Châtillonnais) et montraient que certains gestes féminins jugés mineurs étaient en fait des gestes essentiels pour une culture rurale partagée entre hommes et femmes (la mort du cochon, la broderie du trousseau, etc. furent des exemples de ces études). De même furent étudiés en ce sens les partages spatiaux (la cour, les champs) et les partages domestiques. Dans aucun de ces cas marquants dans l'historiographie féminine ne fut employé le mot consentement. Les études de F. Zonabend et d'Y. Verdier se situaient plutôt dans des hypothèses d'apaisement et d'immobilité fonctionnelle des rôles sociaux de chaque sexe. Un article bilan du groupe d'histoire des femmes ${ }^{19}$, paru dans les Annales, revint et réfléchit sur toutes ces problématiques.

Une nouvelle ère de travaux s'ouvrit sur un pôle inconnu des études fémininesféministes: la prise de pouvoir des femmes. Une fois bien situés les espaces de domination et bien expliqué que le modèle domination-oppression ne pouvait disparaître, l'intérêt se porta sur les moments, les temps, les âges, les situations politiques où des femmes (non pas des femmes exceptionnelles) exercèrent leur pouvoir. Puis, les reines de France ${ }^{20}$, les maîtresses des rois, les princesses furent étudiées sous un autre jour que celui des alcôves. Les religieuses, les béguines ou les mystiques (M. de Certeau) firent l'objet d'ouvrages savants, montrant l'ascension sociale de certaines femmes par ce biais ou l'autorité spirituelle de certains couvents dans la recherche de Dieu. Ajoutons à cela l'étude des formes d'assistance, de philanthropie, du rôle maternel.

De même, certaines situations politiques furent déclinées sous l'angle d'un pouvoir possible, pris de façon évidente, même si momentané : on pense aux travaux sur les émeutières au XVIII ${ }^{e}$ siècle $^{21}$, aux femmes sous la Révolution ${ }^{22}$, à certaines formes de délinquance spécifiques, aux protestantes des refuges cévenols, aux catholiques et protestantes pendant les guerres de religion, aux ouvrières en grève, aux femmes des barricades de 1870, aux femmes pendant les Grandes guerres et, enfin, aux résistantes. Ainsi était-il question de faire l'histoire approfondie de ces îlots où des formes de pouvoir avaient pu être prises, même sur un temps éphémère.

21 Dans ces problématiques, c'était, bien entendu, et cela n'avait rien que de très normal, le non-consentement à l'ordre établi qui régnait. Pourtant, certaines études (les émeutières $d u x_{\text {XVIII }}{ }^{e}$ siècle par exemple) montraient que ce partage différent (femmes en avant dans les émeutes, hommes en arrière) se faisait avec le consentement masculin et ne remettait pas en cause les partages traditionnels.

Quoi qu'il en fut, les grilles de lecture, les modes de réflexion s'affinaient pour approcher de façon de plus en plus subtile des types de réalités qui étaient celles de certaines femmes. Subsistait toutefois l'impossibilité théorique pour la plupart des études féministes d'aller dans des champs un peu plus complexes et ambivalents, où les femmes risquaient d'apparaître sous des jours plus sombres, moins idylliques que ceux 
auxquels les avait destinées une certaine histoire des femmes, militante et combative (cela avec raison).

Les réflexions sur la violence des femmes comme celles sur la séduction ${ }^{23}$ firent effort pour entrer dans des types de rapports de force entre le masculin et le féminin, où les rôles se distribuaient de façon non linéaire, non attendue, parfois transgressive. Admettre que la femme puisse être actrice de la séduction et chercher à comprendre comment l'Eglise, les discours et les médecins ont tenté de décrire, d'imposer cette attitude était déjà très important. Aller plus loin, c'était entrer dans le plaisir de la séduction ressenti par certaines femmes: ici, quelque chose d'un féminisme bon teint vacillait. Plaisir, sensualité, relations entre les corps n'ont pas encore trouvé leurs historiens, même s'ils ont leurs peintres et leurs poètes.

Pour cela, il faudrait travailler sur la notion de consentement. Les femmes, par moments, consentent-elles à leur situation pour ensuite la détourner à leur avantage ? Le débat est sans fin, c'est pourquoi il serait si passionnant de l'amorcer et de poursuivre la réflexion de Nicole-Claude Mathieu ${ }^{24}$. L'histoire des moments sociaux et politiques (voir les études de Pierre Laborie) montre comment les acteurs sociaux et l'ensemble des êtres humains possèdent des pensées doubles, empruntent des chemins où règne l'ambivalence, où marquent par leur silence des formes aussi compliquées que celles qui vont de l'indignation à la stupeur, de la résignation à la compassion. Sans compter les moments où ces formes sont vécues de façon simultanée. Pourquoi les catégories de sexe échapperaient-elles à cette particularité qui a son historicité, ses moments et ses formes propres?

Survint, comme un véritable coup de poing, le livre de Pierre Bourdieu, La Domination masculine ${ }^{25}$. En dehors du fait que ce livre souleva un tollé pour n'avoir cité aucun des travaux féministes et n'avoir que peu tenu compte d'un nombre important d'études et d'ouvrages autour de ces problèmes, les chapitres sur l'intériorisation symbolique des valeurs féminines par les femmes (reprenant le thème cher au théoricien de la violence symbolique) mettaient le consentement féminin au premier plan. Y a-t-il consentement s'il s'agit d'intériorisation de modèles ? Et ensuite, comment et pourquoi prend-on du plaisir à activer ces formes d'attitude?

Au moins, le problème est posé, et s'il est certain que Pierre Bourdieu l'a abordé sur le plan corporel et de la relation singulière homme-femme, il reste une quantité de situations politiques qui demanderaient que soient étudiées des formes de consentement. Le consentement de certaines femmes au nazisme, au racisme, à la violence des génocides ou à celle des massacres pose question. De même - dans un autre registre -, le consentement à la guerre ou, au contraire, au pacifisme. Dans les options politiques des femmes, quelle part faire à des avis marqués du consentement à l'ordre politique masculin si cet ordre est, par exemple, en train de basculer du côté de la transgression ou de la subversion? Les femmes résistantes ont-elles consenti au partage des tâches traditionnelles ou ont-elles inventé, sous couvert de leurs fonctions habituelles, des formes inouïes de résistance?

C'est un questionnement très ample dont il s'agit ici, et il faudrait d'ailleurs aussi étudier les moments ordinaires des consentements quotidiens masculin et féminin, les instants où ils se déplacent et s'inversent. En aucun cas ce thème ne "désacralise " (peur panique d'un certain féminisme) les déterminations féminines de l'émancipation. J'aurais tendance à croire, pour ma part, que c'est dans la matière même des consentements ordinaires qu'un jour se fabriquent les luttes. 
28

\section{Classes sociales}

Au début de l'histoire des femmes, une forme d'étude s'est imposée: celle des conditions féminines. Il fallait éclairer et historiciser ces situations inconnues des historiens et du public où se trouvaient de nombreuses femmes au travail. Ce furent des ouvrages sur la blanchisseuse, la prostituée, la criminelle, la soignante, l'ouvrière à l'aiguille, la nourrice, la religieuse, etc. Un thème majeur fut celui de la domestique, de la servante, du rapport femme-femme qu'on pouvait instaurer. Dans ces études, quelque chose était plus proche de la monographie que d'une réflexion sur les rapports de sexe, plus proche de la description d'une condition dont il était dit à l'avance qu'elle était bien évidemment dominée, que de la discussion de ce concept.

Sans doute cette pratique en vint à s'effilocher non seulement d'elle-même car elle était répétitive, mais aussi face à une réception en milieu universitaire peu favorable : de cet alignement de métiers féminins sans gloire, les pairs ne voyaient que misérabilisme et un certain manque de problématique. L'histoire des femmes leur semblait (à tort) le lieu d'une grande pauvreté intellectuelle.

31 Dans les années 1980, au fur et à mesure des travaux produits, des séminaires mis en place et aussi de la combativité des historiennes pour apparaître historiennes «à part entière ", de nouvelles thématiques apparurent comme : le pouvoir des femmes, les formes de relation entre les sexes, les thèmes complexes de la violence des femmes et sur les femmes. Plus timide fut l'apparition de thèmes concernant des événements. L'histoire culturelle et celle des représentations, si elles apportaient d'intenses éclairages et une nouvelle façon de travailler sur les sociétés, arasaient en même temps les postures conflictuelles, et si la différence des sexes pouvait être mentionnée (dans les études sur la lecture, sur l'écriture par exemple), elle n'était guère mise en avant, simple conséquence du regard global sur le monde des cultures et des représentations. Pourtant les domaines de l'acquisition de la culture étaient drastiquement séparés entre classes sociales. D'ailleurs, après que sont sortis, en 1991, les cinq volumes d'Histoire des Femmes et qu'un colloque s'est réuni à la Sorbonne autour de cette publication, Jacques Rancière, philosophe, sut déjà pointer cette déficience. Quelque chose des différences entre couches sociales, ainsi que de ce qu'il appela « les travaux et les jours ", c'est-à-dire des aspérités du quotidien entre hommes et femmes, lui paraissait avoir été, non oublié dans ces cinq volumes, mais relativement peu abordé.

Bien entendu, ces débats et ces critiques avaient une origine : au début des années du féminisme, et surtout dans les milieux universitaires et politiques qui se créèrent en 1968, une vaste controverse s'organisa immédiatement sur le thème suivant : « la cause des femmes » était susceptible de faire tort à «la cause du/des peuple(s) » et cet argument fort fut combattu en même temps qu'il eut des conséquences «intériorisantes » pour chacune ${ }^{26}$. Des traces de ce débat sont restées; peut-être que pour certains, l'accentuation (bien normale et légitime) sur « la cause des femmes » fit un travail d'oblitération légère de l'affrontement entre classes sociales, dans lesquelles les femmes à la fois tiennent des rôles et sont prisonnières de rôles ${ }^{27}$.

Réfléchir à cette relation entre histoire des femmes et problématique des classes sociales permet d'expliciter ce qui fut vécu comme un malentendu. Il est urgent d'en démêler les fils au moment où le terme « classe ouvrière » disparaît du discours et des préoccupations politiques, au moment où se brouille l'horizon féministe. Pour cela on peut distinguer quatre filtres à travers lesquels penser cette situation : 
34 Malgré des études ayant mis en lumière certaines figures féminines populaires (émeutières, grévistes, ouvrières « ovalistes »...), la présence de la composante féminine active, pourtant si évidente, au sein des classes populaires et ouvrières est restée dans l'historiographie relativement invisible ${ }^{28}$.

35 L'écriture de cette histoire, longtemps inspirée par des paradigmes marxistes, a «omis » de parler des femmes, pour des raisons idéologiques ou pratiques. On n'a donc pas cherché à les voir dans des archives et des institutions construites au masculin. On n'en finit pas de solder cet héritage d'effacement redoublé par une vision éminemment économiste et comptable du travail (d'elles, il est toujours peu parlé dans les grandes synthèses d'histoire du travail).

36 Le mouvement féministe des années 1970 comme celui de la fin du XIX ${ }^{\mathrm{e}}$ siècle, est sociologiquement issue des classes moyennes ou de l'élite instruite. Raison spécieuse ou circonstancielle, l'origine sociale du chercheur peut-elle constituer un handicap, ou un péché d'ethnocentrisme qui l'empêcherait de se soucier des autres? Il importe de distinguer les tensions sur le terrain politique et syndical, les affrontements effectifs entre féministes et ouvriers à des moments précis de l'histoire, et le monde de la recherche qui a précisément lié l'histoire des femmes et la cause des « oubliés ».

Ce champ qui s'est peu à peu développé a bien pointé que des pans entiers des recherches ont redoublé la construction historique du monde ouvrier au masculin neutre. Ainsi les figures du "mineur musclé », les catégories d'ouvrier, de cadre, de travailleur ... construites en rupture avec la vie hors travail, les critères de la qualification fondés sur la force, la notion de "ménage " en référence au père ou au mari, la mesure de la pauvreté, la vision des règles et des pratiques des corporations, ou du syndicalisme... cette conjugaison du travail au masculin appartient à un passé dont nous avons hérité et dont il importe justement de questionner la neutralité.

Il faut dire que la conjoncture sociale et politique a renforcé le mouvement: les idéologies ayant été considérées comme mortifères pour la vie des sociétés, les prismes intellectuels furent teintés d'un affaissement des concepts offensifs, ou d'un vocabulaire plus positiviste, donnant aux choses et aux situations leur affectation précise.

39 Dans l'introduction de son livre Les Femmes dans la société française, $\mathrm{XVI}^{e}-\mathrm{XVII}{ }^{e}$ siècles ${ }^{29}$, Dominique Godineau écrit : «Il faut insister sur la diversité des vies, des conditions, dire et redire que les femmes ne forment pas une population homogène sans attache sociale ou chronologique, qu'elles appartiennent parfois à des groupes antagonistes. Dire aussi que ce n'est pas parce qu'elles sont femmes qu'elles sont nécessairement solidaires. Gestes d'entraide et paroles réconfortantes face au « malheur d'être femme » dans une société injuste et inégalitaire voisinent avec rivalités, mépris social ou haines religieuses [...] N'oublions jamais qu'il s'agissait de personnes réelles, et que l'existence ne peut se réduire aux normes imposées, que les rapports humains sont faits de révoltes individuelles, d'arrangements, d'accords, de tractations. »

Les femmes sont des "personnes réelles"; l'enjeu est là, et il y eut de véritables travaux sur cette réalité. Par exemple, l'histoire de la question sociale et de sa régulation par l'État depuis le XIX ${ }^{\mathrm{e}}$ siècle, bien étudiée ${ }^{30}$, montre que cette histoire est inséparable de la conception que la société se faisait de la différence des sexes. Ainsi fut-il bien mis en avant que la présence des femmes sur tous les lieux de production ou de service posait un problème dans l'organisation du travail (imposant une redéfinition 
des espaces et des frontières entre les sexes : entrées séparées dans les banques et les grands magasins, tenues vestimentaires réglementées, etc.). Le travail féminin ne faisait pas seulement concurrence aux travailleurs mais il menaçait la vie de la famille, la natalité et donc la nation. Les mesures de protection pour elles, les lois en leur faveur (réduction du temps de travail, interdiction du travail de nuit, puis congés de maternité) en vinrent de fait à créer de lourdes formes d'exclusion. Le travail des femmes était alors perçu comme une réelle difficulté dans les esprits des élites et des politiques, dont il subsiste encore des traces aujourd'hui.

On pourrait, bien sût, prendre d'autres exemples que celui des formes de protection sociale et s'appuyer sur d'autres époques : ainsi au XVIII siècle, la femme de l'artisan, totalement inscrite dans les faits du monde du travail, préfère échapper aux réglementations des corporations pour tenir un rôle, certes diffus mais puissant, auprès des compagnons et des apprentis et même de sa famille. Situation dont le mari sait tirer parti à maintes occasions.

On le voit, ce thème femmes-travail-classes sociales est très producteur pour repenser la question sociale, l'Etat providence, l'économie du travail. S'il importe de " comprendre de quelle manière les classes sociales sont affectées par le fait qu'elles englobent deux sexes " (Joan Scott), il est aussi urgent d'inscrire les questionnements sur l'histoire des femmes dans la pluralité et la complexité des attaches sociales, religieuses et culturelles. Il faut bien constater que ces perspectives de nomination des différences sexuelles face à des situations particulières de rapports de classes ou de travail ont peine à devenir des modèles d'interprétation communs à l'ensemble de l'histoire sociale.

Dans les ouvrages écrits sur le travail des femmes, les données ont parfois été croisées avec la possibilité ou l'impossibilité pour les femmes de gérer le travail domestique; pourtant il pourrait y avoir des nouvelles avancées auxquelles il ne fut pas encore songé. Il serait très novateur et intéressant par exemple, de prendre le parti de relier intimité et vie du travail dans les siècles précédents ; cela permettrait, entre autre, de renouveler une histoire des corps qui s'échapperait alors des savoirs traditionnels transmis sur la question et ferait entrer dans une histoire sociale peu explorée, celle des intériorités masculine et féminine, à travers les classes, mais aussi selon les moments, époques, situations et événements.

Croiser le désir, la passion, la séduction avec les règles et les formes du travail en tenant compte des événements sociaux et économiques et des groupes sociaux serait un champ neuf susceptible d'apporter beaucoup non seulement à l'histoire des femmes, mais à celle d'aujourd'hui. Des siècles, comme ceux de l'Ancien Régime ou du XIX ${ }^{e}$ siècle peuvent bien évidemment supporter cette grille de lecture, car la question de l'intériorité des sentiments face aux manières de partager le travail ou de dominer celui des femmes, a toujours rejailli aussi sur les formes de subversion, de conflits.

Certaines situations comme la disette ou les guerres sous la monarchie, les conditions de vie ouvrière au XIX ${ }^{e}$ siècle, les révolutions (1848, la Commune), le chômage et la précarité ont eu et ont beaucoup de conséquences non seulement sur les rôles entre les sexes mais sur les représentations que chacun des sexes a de l'autre. D'autres temporalités s'organisent en même temps que des formes d'attente, et chaque événement transforme l'ordre des sentiments et des constructions du partenariat. Les corps féminins et masculins apprennent de nouveaux gestes, soit dans la séparation, soit à travers les accidents du travail, soit dans la guerre, soit dans les crises politiques. 
Et toute une alchimie de passions intimes, sociales et politiques s'organise et se réorganise, et se décline selon les niveaux sociaux.

L'histoire de la construction entre les sexes face à de telles interrogations croisant le corps, le travail, les classes sociales et les cultures amoureuses qui en dépendent pourrait être urgente à entreprendre.

\section{C.D. et A.F.}

Éclats de paroles et moments de silence

istoire des femmes restent des silences ${ }^{31}$, des silences troués de voix individuelles et collectives, de cris et de murmures, de protestations et de harangues. Comment furent perçus ces éclats de parole des femmes de jadis? Le statut accordé à la parole des femmes par les scientifiques semble indissociable de la place que cette parole occupe dans la société contemporaine. À un moment (les années 1970) où paraissent une multitude éphémère de journaux qui ont pour titre Les Mûres prennent la parole, Parole, Les Femmes s'entendent (Besançon), La Voix des femmes (Lyon), etc. les historien(ne)s ne peuvent s'abstraire d'une attitude plus large de la société et actualisent dans leur discipline le statut accordé par les médias à la parole féminine. Les femmes en lutte font reculer l'amnésie.

En quelques décennies, le monde universitaire s'ouvre discrètement aux femmes ; et les femmes deviennent des sujets d'études légitimes ${ }^{32}$. La fragilité de cette reconnaissance et la nécessité de défendre en permanence l'existence de ce domaine n'ont pas mis obstacle - et même d'une certaine façon ont contraint - à la constitution d'un champ propre à l'histoire des femmes. La concomitance entre la prise de plume des unes et l'écoute accordée aux femmes est soulignée par les historiennes: "l'histoire des femmes est, d'une certaine façon, celle de leur prise de parole ${ }^{33}$. Pas plus qu'elle ne s'écrit à l'écart du monde, l'histoire des femmes ne se développe de façon autonome ; elle est portée par le même mouvement que les autres sciences sociales, attentives aux «façons de dire $»^{34}$ et aux « dires de femmes » ${ }^{35}$.

Parole vive et mémoire

La pratique de l'histoire requiert des sources : la «nouvelle histoire » des années 1970, en annexant des territoires sans cesse plus nombreux, a sollicité des documents très divers, des sources négligées, invisibles et muettes auparavant. Parmi un ensemble de matériaux déposés au cours des temps sous forme de traces, de pratiques, d'institutions ou d'œuvres, la parole des femmes a émergé comme source et comme objet de recherche - l'écho de la parole plutôt, puisqu'elle ne reste accessible qu'à travers une transcription écrite qui impose ses formes. On se met à l'écoute sur tous les fronts, de l'aristocrate célébrée à la prolétaire analphabète : femmes émeutières, militantes ou grévistes, membres d'associations et médiums, témoins de rixes et salonnières. Il faut mentionner l'invite antérieure de Lucien Febvre, préoccupé il est vrai d'histoire politique et culturelle et de processus sociaux et non de relations entre les sexes, de porter attention à la linguistique, aux mots, aux langages populaires sous forme écrite ou orale.

51 Dans leur champ propre, les historiennes des femmes ont d'abord mobilisé la parole autour de la question de la mémoire. Elles ont longtemps pensé que les archives étaient très ténues. Alors que les femmes revendiquent un droit d'expression dans la presse, la rue et les réunions, il a paru urgent et nécessaire de rechercher le témoignage des femmes. Les enquêtes orales permettent d'accéder aux faits de la vie quotidienne, à un moment où il est affirmé que le privé est politique, et devient objet d'histoire. Les 
recherches s'inscrivent dans le courant de l'histoire orale, qui puise dans la mémoire propre des oubliés, des ouvriers, des marginaux, etc., et qui, rendant à chacun sa place d'acteur, les fait advenir à l'existence. Les témoignages ne sont pas cités en vignettes illustratives, mais sollicités pour présenter des expériences inédites, prolonger le regard, renvoyer à d'autres interrogations, parfois laisser entendre ce qui ne se savait pas - on songe aux recherches sur le corps, les lesbiennes, les femmes dans la guerre.

Exemplaire de ce courant, la revue Pénélope, «libre tribune permanente sur les problèmes que posent l'histoire et l'anthropologie des femmes, leurs sources nouvelles, leurs méthodes spécifiques, les voies de recherche défrichées par des publications récentes", qui se veut attentive aux "tentatives d'histoire orale, où les femmes, souvent gardiennes de la mémoire, devraient avoir tant de place ", et pratique "la quête des souvenirs les plus quotidiens des mères et des grands-mères, irremplaçables témoins d'une histoire non écrite ${ }^{36}$. Ce n'est pas un hasard si le premier numéro de cette revue de la recherche militante est consacré à la presse féminine et féministe depuis le XVIII ${ }^{\mathrm{e}}$ siècle et met en lumière « la lutte perpétuellement recommencée pour la parole des femmes et la survie des journaux $\|^{37}$.

Retrouver les mots des femmes

Il apparait vite que les mots des femmes du passé sont recueillis par des hommes. Ce sont eux qui le plus souvent ont mis en forme la parole des femmes et rédigé les documents qui les concernent, eux qui ont compté et enregistré, eux qui ont opéré tris et classements, constitué les archives, imposé les catégories d'interprétations, dit ce que doit être, dire et faire la Femme. La Femme écrase de sa majesté abstraite et idéalisée les femmes plurielles, réelles. Dans les discours, les images priment sur les informations concrètes: les femmes sont représentées avant d'être décrites ou racontées et «l'enregistrement primaire de ce qu'elles font et disent est médiatisé par les critères de sélection des scribes du pouvoir. Indifférents au privé, ils s'attachent au public où elles n'entrent pas. [...] Le rapport des sexes imprime sa marque aux sources de l'histoire, et conditionne leur inégale densité. $\rrbracket^{38}$ De la même façon que les mots des marginaux qui gisent dans les archives nées de la répression témoignent d'abord sur la norme imposée ${ }^{39}$, les mots des femmes échappés au silence tracent en creux les contours d'une société structurée par des figures masculines de pouvoir.

Lorsque les femmes s'expriment directement, leur parole subit la contrainte de modes contrôlés par les hommes : non confisquée, elle est cependant formatée, élaborée dans l'ombre des discours de théologiens, de médecins, de pédagogues, de juristes, de moralistes, d'hommes de lettres, de savants, d'éditeurs, etc. Les dominants imposent à tous leurs cadres de pensée en mobilisant les ressources du langage, et la parole des femmes se trouve prise dans la problématique du rapport des sexes et de la domination masculine. Même le courant saint-simonien qui se prononce pour l'égalité sociale des sexes et proclame "Femme! le monde a besoin de ton verbe ${ }^{40}$ dépouille le désir féminin de son expression propre et le plie à sa rhétorique ${ }^{41}$. Il paraît alors nécessaire de rechercher, au-delà de l'imaginaire masculin et de son autorité, des faits matériels et sociaux, qui disent le réel, les formes quotidiennes, la vie des femmes avec leurs mots, reflets d'une « culture féminine » spécifique.

Recherche identitaire

55 Le troisième moment, après le repli sur la voix mémorielle et le détour par la critique des interventions masculines, opère un retour aux discours des femmes - sur ellesmêmes et, de plus en plus, sur la sociététe ${ }^{42}$. La parole vive est restée longtemps inaudible 
parce que souvent peu structurée, proche de l'expression orale, discontinue et fugitive. Surtout, on ne voulait ou pouvait pas l'entendre ${ }^{43}$ : la spontanéité est subversive. Prendre la parole représente une des modalités de la critique ou de la résistance à la domination - et la contestation du pouvoir est souvent dépréciée, dénigrée, occultée. Liée aux situations de crise, la parole politique rejetée par les hommes et les milieux au pouvoir (pensons aux mouvements sociaux et aux grèves surtout lorsqu'ils échappent au contrôle des syndicats), peu entendue sur le moment, très vite oubliée ensuite, se repère pourtant dans des tracts, des pétitions, des déclarations, des brochures, des journaux, des discours manuscrits ou imprimés, des écrits personnels, etc. Le travail des historien(ne)s s'oriente donc vers la recherche de l'expression des femmes, son écoute et l'analyse de ses conditions de production et d'ensevelissement.

Les intérêts et fascinations que suscitent les paroles de femmes rejoignent ceux que déclenchent les "paroles de détenus ", ou les "paroles de poilus " qui sont recueillies et portées à la connaissance du public. Ces contrepoints aux discours dominants portent les questions du statut des témoignages, de leur élaboration et de leur " découverte ", de l'intelligibilité de ces traces sensibles et précaires, du sens collectif à leur donner au-delà de leur singularité. Il revient à un patient travail d'interprétation, de confrontation, de recoupement, de critique et d'analyse, de dépasser l'illusion de vérité des mots retrouvés et de restituer aux paroles brutes leur dimension sociale, politique - et historique. Ces paroles inédites, resituées dans une trame déjà constituée, permettent alors d'interroger le partage d'un passé national commun et la mixité de la mémoire, le rapport entre pratiques sociales et représentations, la surdité récurrente de ceux qui écrivent l'histoire et les mirages de leurs lectures.

Les groupes de femmes que structurent les mots échappés au silence, comme les autres ensembles sociaux dont on sait bien qu'ils ne sont pas des entités fixes, se construisent en essayant d'échapper aux relations de pouvoir fixées dans le langage. Plus peut-être que pour d'autres catégories, la parole des femmes - sœur jumelle de l'écriture apparaît liée à une quête identitaire et au désir d'exister. Un désir si dérangeant que des hommes à leur tour, bousculés par des expressions qu'ils (dis)qualifient de féministes, cherchent à opposer leurs propres groupes de paroles. Dans le tumulte confus où s'affrontent tant de voix diverses qui sans cesse courent le risque d'être recouvertes ou confisquées par une communauté, un rassemblement d'intérêts, un parti, il reste difficile à une parole féminine d'émerger, vigilante et légitime, écoutée et entendue.

D.P.

À propos de « l'écriture féminine »

59 «L'écriture féminine " a constitué un point d'ancrage fort pour le mouvement et les études féministes des années 70 . La prise de conscience identitaire a été inséparable d'une mise en scène de la parole, de l'écriture, du rapport au langage et au discours. L'urgence était de dénoncer le codage des positions d'énonciation, les modalités et les contenus des discours qui sont au cœur du fonctionnement symbolique et du système patriarcal ${ }^{44}$. Dans le domaine littéraire et plus généralement artistique, cette revendication s'est déployée dans de multiples directions pour tenter de définir et d'imposer une «identité - unité artistique collective liée à la différence des sexes »"

Dans cet épisode que certains ont assimilé à une conquête ${ }^{46}$, on peut retenir quelques traits majeurs, notamment la récurrence de thématiques "existentielles », la quête d'une nouvelle identité, d'un nouvel espace de liberté où les femmes ne seraient plus 
perçues comme l'Autre, comme non-identité, non-entité, non-unité (par rapport à l'Un du Père, de l'Homme, du Phallus) mais justement comme différence. Pour Luce Irigaray qui porte haut et fort la théorisation de la différence dans et par l'écriture, la femme existera vraiment le jour où son corps "parlera sa spécificité ». Ainsi se noue un lien logique et explicite entre la différence inscrite dans le corps et la recherche d'autres modes d'expression. La narration féminine (analyse menée dans l'ouvrage De Piaggi à partir d'une centaine de romans) se caractériserait par ses structures temporelles (par exemple dans l'emploi fréquent de flashback, la démarche circulaire de la mémoire, la propension à l'errance), par l'observation de l'univers sensible (qui établit une correspondance entre l'intrigue et l'espace imaginaire, entre la vie psychologique des personnages et le milieu ambiant), par la symbolisation d'espaces clos comme le jardin ou d'éléments comme le sang et l'eau, et par l'utilisation des facultés sensitives (au plus près des émotions et des frémissements qui donnent accès au refoulé). Ces traits ont parfois été poussés à leur paroxysme, jusqu'au repliement de l'auteur sur sa subjectivité, jusqu'à l'assimilation du féminin au maternel, usant et abusant de la métaphore naturalisante du fait politique de la domination. Ecriture et féminité ont été associées pour désigner une production biologique, une sécrétion naturelle, une spécificité corps/femelle/nature. "L'écriture féminine " échappait ainsi à l'histoire, enfermée dans le symbolique et l'invariant ${ }^{47}$.

61 Il est important de souligner que la thématique de "l'écriture féminine » occupait surtout le terrain féministe et littéraire. Dans la discipline historique, elle émerge à travers le concept de «culture féminine», et sous l'influence de travaux ethnologiques ${ }^{48}$. Ecriture et culture, dites féminines, ces deux concepts semblent céder à la même tentation qui consiste à découper un espace, des objets et des pratiques dans le monde social qui appartiendraient en propre aux femmes et à les qualifier de féminins. Le concept de " culture féminine " a été critiqué dans l'article des Annales de $1986^{49}$, puis récusé par Agnès Fine ${ }^{50}$. Au moins pour deux raisons principales. En premier lieu, les cultures et les représentations du féminin qu'elles portent, au-delà des modalités d'expression, d'incorporation et de transmission, sont partagées par les hommes et par les femmes. Elles énoncent des systèmes d'intelligibilité cohérents et nécessaires à tous les acteurs sociaux. Deuxièmement, la culture, qu'elle soit qualifiée de féminine ou masculine, de populaire ou savante, est toujours le produit d'une relation de pouvoir. Elle se décline à l'intérieur de rapports inégalitaires, en masque les failles, réactive les conflits, jalonne temps et espaces, et s'applique à conceptualiser et à mettre en scène ses particularités et ses relations avec la société globale.

Le contexte historiographique des années 80 a suscité un pivotement de point de vue : "l'écriture féminine " ne fut plus appréhendée pour elle-même, dans son "essence ", mais en rapport avec les conditions de sa production et de sa réception. On peut observer ce déplacement dans deux champs distincts mais souvent croisés : l'histoire littéraire, fortement influencée par la critique post-moderniste venue des États-Unis, et l'histoire sociale de la culture.

63 En histoire littéraire, de nombreux travaux ont pu faire émerger des figures féminines, restées dans l'angle mort du passés1. Ils ont été confrontés aux malentendus de l'idéologie qui classe et hiérarchise les genres textuels, et aux clichés trop confortables de la féminité qui ont englouti les femmes auteurs dans les marges de la critique, hors des lieux de légitimation et des instances de canonisation. La recherche semble buter sur les processus qui font que les écritures produites par des femmes se sont 
développées dans des genres dits mineurs, et que, conjointement, la qualification par le féminin contribue à hiérarchiser les genres. L'histoire du genre épistolaire semble bien illustrer ce processus ${ }^{52}$. Elle suggère que le champ littéraire s'est construit durablement en négligeant la production féminine, ou plutôt que la marginalisation d'une écriture manuscrite et d'une culture orale a pu se conjuguer avec les procédures de hiérarchisation et les rapports de force qui ont façonné cet espace. Il reste encore à clarifier la construction des catégories du masculin et du féminin dans les pratiques d'écriture, dans l'élaboration des œuvres comme dans leur réception. Il s'agirait alors moins d'affecter des genres (mineurs), des rôles (de muses ou collaboratrices), des thématiques (religieuses ou intimes), ou encore des qualités (comme la spontanéité ou le naturel), à des auteurs classés en fonction de leur identité sexuée, que de repérer comment la création littéraire absorbe l'un et l'autre sexe en déplaçant sans cesse les lignes de partage, en mettant en œuvre des composantes affectives, cognitives et émotionnelles autant que savantes et techniques. De nouvelles perspectives pourraient s'ouvrir avec l'examen de cette construction paradoxale des genres dans la fabrique d'une littérature qui ne peut être désincarnée, ni désengagée des conditions pratiques de son élaboration.

Les problématiques de l'histoire sociale de la culture ont également contribué à renouveler le questionnement sur "l'écriture féminine». Le concept des «écritures ordinaires ", proposé par les anthropologues ${ }^{53}$, a fait éclater le corpus des œuvres préconstruit, les fonctions, les usages sociaux et symboliques d'une multitude de formes écrites, considérées indépendamment de leur valeur esthétique. Cette approche invite à déplacer le point de vue de l'œuvre vers le travail et la prise d'écriture, de l'énoncé vers l'énonciation, de l'objet écrit vers l'acteur, vers ses stratégies de conquête et d'appropriation. Les années 90 ont vu s'ouvrir un vaste chantier sur les archives privées, les correspondances, les journaux intimes, les mémoires et les autobiographies. Après une phase de sacralisation, dans une conception plutôt militante de l'histoire soucieuse de faire entendre les marginaux et les exclus, les écrits "perdus " puis "découverts » ont fait l'objet d'enquêtes sur les conditions de leur production, sur toute l'épaisseur des expériences quotidiennes dont ils témoignent, sur les effets de sens induits par leurs formes matérielles et leurs modes de transmission. Dans nombre de ces travaux, la question du genre est présente. Souvent posée en termes de spécificités, elle s'efforce - encore insuffisamment - d'inscrire les pratiques et les représentations dans un continuum social et culturel. Elle est notamment confrontée au poids de l'injonction visant les femmes à tenir les écritures domestiques ${ }^{54}$. On peut encore s'interroger sur les modalités de l'intériorisation ou de soumission à cette injonction. Il s'avère en tout cas que la valorisation des ego-documents et des expressions individuelles ne peut faire abstraction des cadres sociaux qui les portent, de ses clivages et de ses effets de distinction.

Autrement dit, la quête identitaire par l'écriture a effectivement une histoire. Le mouvement féministe des années 70 en marque un moment fort, une sorte de "retour » sur la scène politique dont les spécialistes de l'histoire du saint-simonisme n'ont pas été dupes ${ }^{55}$. Les "saint-simoniennes" ont été considérées comme des précurseurs du féminisme contemporain, auxquelles les universitaires américaines ont été tentées de s'identifier: "comme nous » elles ont été dans et hors l'institution, " comme nous» elles ont fondé leur analyse de l'oppression socio-économique sur la répression du corps féminin, « comme nous » elles ont valorisé les qualités féminines 
comme source régénératrice, "comme nous » enfin elles ont eu recours à l'écriture comme stratégie de libération.

"Paroles de femmes », "écriture féminine » pour dire la différence, les expressions favorites des féministes radicales de la fin $\mathrm{du} \mathrm{xx}^{\mathrm{e}}$ siècle scandaient déjà la production saint-simonienne. Il est frappant que dans les deux cas la figure maternelle soit posée comme une alternative au monde cruel et menaçant où règne la Loi du père. En rapprochant ces deux temps forts de la présence féminine sur la scène politique, il devient évident que la thématique écriture et parole féminine a partie liée avec celle de la maternité. L'une et l'autre émergent, dans les années 1970 comme à l'époque du Saint-Simonisme, dans la foulée de mouvements sociaux et politiques. L'une et l'autre occupent une place significative dans la revendication identitaire. Simple concomitance ? Faire ce rapprochement ne vise pas tant à chercher une similitude dans les trajectoires qu'à pointer leur enracinement dans l'ordre existentiel, du moins perçu comme tel quand les femmes en viennent à questionner la construction sociale de la différence entre les sexes et les valeurs qui justifient la division du travail et le système symbolique $^{56}$. Créer et/ou procréer, la question est effectivement existentielle ${ }^{57}$.

Bousculant les stéréotypes de l'éternel féminin et de l'instinct maternel, les historiennes, dès leurs premiers travaux, ont délibérément saisit le thème de la maternité pour en décliner l'historicité, pour souligner les enjeux sociaux et politiques de l'acte de donner la vie, et non pas seulement démographiques ou médicaux ${ }^{58}$.

La maternité a certes accédé au statut d'objet historique, elle n'en demeure pas moins une question existentielle et, sans doute, une affaire de génération. Les femmes qui militent dans les années 70 pour le droit à la contraception, à l'avortement, à la libre disposition de leur corps sont filles du baby-boom. Au-delà des appartenances sociales, ces féministes se démarquent de leurs aînées qui, elles, ont vécu ou subi, durant l'Entredeux guerres, l'exaltation de la mère au foyer, pilier de la nation et gardienne de la moralité. La "génération 68 " bénéficie de la progression des études, de la mixité toute récente dans les écoles et compte de plus en plus de diplômées. Elle profite aussi des Trente glorieuses, d'un marché plus favorable et plus ouvert au travail des femmes. Féministes et historiennes des années 70, dans leur double engagement, expérimentent une palette inédite de choix de vie tout en étant projetées au cœur de bien de contradictions : entre ce que leurs mères et la société leur ont transmis, et ce que le contexte politique permet d'envisager. La possibilité même de pouvoir choisir (dans l'idéal) ouvre contradictoirement la voie du refus de la maternité ressentie comme un piège, comme une aliénation, et la voie de l'exaltation d'une nature spécifique, valorisée. Être ou ne pas être mère, la question devient le creuset d'une mutation où peuvent à la fois s'épancher la spécificité féminine, se déverser l'expression de rêves et de désirs, et s'affirmer un choix individuel comme acte social.

Après le temps infini du "silence " des mères et après la pléthore des discours tenus sur elles par les ecclésiastiques, les médecins et les hommes politiques, la possibilité d'envisager un autre destin que celui de la maternité, ou de choisir le moment et les modalités de la procréation ouvre effectivement bien des horizons dans tous les domaines mais tend aussi à brouiller les repères d'antan, à déplacer les frontières entre les sexes. Au point de menacer l'empire masculin? Rien ne laisse présager un séisme du côté du système politique et des réseaux de décision qui semblent résister durablement au partage des pouvoirs avec les femmes. Seulement l'expression d'un bouillonnement, de craintes, de fantasmes ou autres mises en garde ${ }^{59}$. À différentes reprises, il s'est 
avéré que les "acquis", encore inexistants dans certains pays (droits à la contraception, à l'avortement et au libre usage de son corps) sont fragiles et que la liberté des femmes par rapport à la maternité est susceptible d'être utilisée à des fins politiques. Conditions économiques, contraintes sociales, inertie des mentalités et vieux fantasmes patriarcaux qui cherchent à contenir l'exorbitant pouvoir de procréer limitent bien souvent la portée de ces acquis.

C.D.

La seconde partie de l'article sera publiée dans le prochain numéro.

\section{BIBLIOGRAPHIE}

Cité, 2003, « Politique de la pornographie », Paris, PUF.

CLIO, Histoire, Femmes et Sociétés, 2002, 16, « Parler, chanter, lire, écrire ».

Cosmopolitiques, 2003, « Ce sexe qui nous dépasse », Paris, Ed. de l'Aube.

Mouvement social, 2002, janvier- mars, Paris, Les Editions de l'Atelier.

Pénélope. Pour l'histoire des femmes, 1979, « Les femmes et la presse », nº 1.

ALBERT Jean-Pierre, 1993, « Ecritures domestiques », in D. Fabre, Ecritures ordinaires, op.cit., pp. $37-94$.

AUSLANDER Leora et ZANCARINI-FOURNEL Michelle (éd.), 1995, Différence des sexes et protection sociale (XIX ${ }^{e}-X X^{e}$ siècles), Saint-Denis, Presses universitaires de Vincennes.

AUTAIN Clémentine, 2001, Alter égaux, Invitation au féminisme, Paris, Robert Laffont.

BADINTER Elisabeth (éd.), 1989, Paroles d'hommes, 1790-1793, textes choisis, Paris, POL.

,- 2003, Fausse route, Paris, Odile Jacob.

BARD Christine, 2001, Les femmes dans la société française au XXe siècle, Paris, Armand Colin.

BAUDRY Patrick, 2001, La pornographie et ses images, Paris, Ed. Agora Pocket.

BOTTE Roger, 2003, « Traite des êtres humains et esclavage, du Congrès de Vienne (1815) au

Protocole de Palerme (2000) : les réponses du droit », La Pensée, 336.

BOURDIEU Pierre, 1998, La domination masculine, Paris, Seuil.

BOUZAR Dounia et KADA Saïda, 2003, L'une voilée, l'autre pas, Paris, Albin Michel.

BULCIOLU Maria Teresa, 1980, L'école saint-simonienne et la femme, Pise, Goliardica.

CERTEAU Michel de, 1980, L'invention du quotidien, Paris, Union générale d'Éditions.

CORBIN Alain, 1984, " Le sexe en deuil et l'histoire des femmes au XIX ${ }^{\mathrm{e}}$ siècle ", in Michelle Perrot (dir.), Une histoire des femmes est-elle possible?, Marseille, Rivages, pp. 102-154.

COSANDEY Fanny, 2000, Reine de France, symbole et pouvoir, XV'-XVIII ${ }^{e}$ siècles, Paris, Gallimard. 
DAUPHIN Cécile et al., 1986, « Culture et pouvoir des femmes : essai historiographique », Annales ESC, 41, pp. 271-293.

DAUPHIN Cécile et FARGE Arlette (dir.), 1997, De la violence et des femmes, Paris, Albin Michel.

DAUPHIN Cécile et FARGE Arlette (dir.), 2001, Séduction et sociétés, approches historiques, Paris, Seuil.

DAVIDOFF Leonore, 1995, Worlds Between : Historical Perspectives on Gender and Class, New York, Routledge.

DELPHY Christine, 1996, L'ennemi principal, 1- Économie politique du patriarcat, Paris, Nouvelles Questions féministes.

-, 2001, Penser le genre, Paris, Syllepse.

DEUDON Catherine, 2003, Un Mouvement à soi, Images du mouvement des femmes, 1970-2001, Paris, Syllepse.

DIDIER Béatrice, 1981, L'écriture-femme, Paris, PUF.

DIJK, Susan van et al. (éd.), 2001, Writing the history of women's writing. Toward an international approach, Amsterdam.

DOWNS Laura Lee, 2002, L'inégalité à la chaîne, Paris, Albin Michel.

DUBY Georges et PERROT Michelle (dir.), 1991, Histoire des femmes, Paris, Plon, 5 vol.

DUFOURMANTELLE Anne, 2003, Sexe et philosophie, Paris, Calmann-Lévy.

FABRE Daniel (dir.), 1993, Écritures ordinaires, Paris, P.O.L.

-, 1997, Par écrit. Ethnologie des écritures quotidiennes, Paris, Éditions de la Maison des sciences de l'homme.

FARGE Arlette, 1991, «Évidentes émeutières », in DUBY, 1991, T. 3, pp. 481-496.

FINE Agnès, 2000, « Écritures féminines et rites de passage », Communications, 70, pp. 121-142.

-, 2002, « Histoire des femmes et anthropologie des sexes. Poursuite du débat ouvert en 1986 », CLIO, Histoire, Femmes et Sociétés, 16, pp. 145-166.

FOUCAULT Michel, 1984, L'usage des plaisirs, Paris, Gallimard.

FRADER Laura L. et ROSE Sonya O. (eds), 1996, Gender and Class in Modern Europe, Ithaca, Cornell University Press.

FRAISSE Geneviève (éd.), 1989, Opinions de femmes, de la veille au lendemain de la Révolution française, Paris, Côté-Femmes éditions.

GODINEAU Dominique, 1988, Citoyennes Tricoteuses. Les femmes à Paris pendant la Révolution, Aix-enProvence, éd. Alinea.

-, 2003, Les femmes dans la société française, XVI ${ }^{e}-X V I I I^{e}$ siècle, Paris, A. Colin.

GOLDBERG Steven, 1973, Inevitability of Patriarchy, New York, William Morrow \& co.

GOLDBERG MOSES Claire et WAHL RABINE Leslie, 1993, Feminism, Socialism and French Romanticism, Bloomington and Indianapolis, Indiana University Press.

GUBIN Eliane et al., 2004, Le siècle des féminismes, Paris, Les Éditions de l'Atelier/ Éditions Ouvrières. 
HAASE-DUBOSC Danielle et VIENNOT Eliane, 1991, Femmes et Pouvoirs sous l'Ancien Régime, Paris/ Marseille, Éditions Rivages.

HAASE-DUBOSC Danielle et al. (éd.), 2002, Enjeux contemporains du féminisme indien, Paris, Edition de la Maison des sciences de l'homme.

HÉRITIER Françoise, 1996, Masculin/féminin. La pensée de la différence, Paris, Odile Jacob.

-, 2002, Masculin/féminin. Dissoudre la hiérarchie, Paris, Odile Jacob.

HESSE Carla, 2001, The other Enlightment. How French Women Became Modern, Princeton university Press.

HIRATA Helena et al., 2002, Dictionnaire critique du féminisme, Paris, PUF.

IACUB Marcela, 2002, Qu'avez-vous fait de la libération sexuelle, Paris, Flammarion.

KLEJMAN Laurence et ROCHEFORT Florence, 1987, L'Égalité en marche. Histoire du mouvement féministe en France , 1869-1914, thèse de doctorat sous la direction de Michelle Perrot, université Paris VII, (publication 1989 : L'Égalité en marche. Le féminisme sous la Troisième République, Presses FNSP/des femmes.)

KNIBIEHLER Yvonne et FOUQUET Catherine, 1980, Histoire des mères du Moyen Âge à nos jours, Paris, Montalba.

LAHIRE Bernard, 1997, « Masculin-féminin. L'écriture domestique », in D. Fabre (dir.), Par écrit, op. cit., pp. 145-161.

LAUFER Jacqueline et al., 2003, Le Travail du genre, les sciences sociales du travail à l'épreuve des différences de sexe, Paris, La Découverte/Mage.

MAGLOIRE Franck, 2002, Ouvrière, Éditions de l'Aube.

MARINI Marcelle, 1991, « La place des femmes dans la production culturelle », in DUBY, T. 5, pp. 275-296.

MARZANO Michaela, 2003, La pornographie et l'épuisement du désir, Paris, Buchet-Chastel.

MATHIEU Nicole-Claude, 1985, L'arraisonnement des femmes, Paris, EHESS.

MC ELLROY Wendy, 1995, A Women's Right to Pornography, NY, St Martin's Press.

OGIER Ruwen, 2003, Penser la pornographie, Paris, PUF.

OGIVIE, 2002, Pro-manifesto, Paris, Flammarion.

PERROT Michelle, 1998, Les femmes ou les silences de l'histoire, Paris, Flammarion.

PIAGGI Giorgio de, 1993, La conquête de l'écriture ou une saison d'écriture narrative au féminin : les années 70 , Fasano, Schena et Paris, Nizet.

PLANTÉ Christine, 1989, La petite sœur de Balzac, Paris, Seuil.

PLANTÉ Christine (dir.), 1998, L'épistolaire, un genre féminin ?, Paris, Champion.

RÉGNIER Philippe, 1998, « Les lettres de Clorinde Rogé à Enfantin » in Christine Planté (dir.), L'épistolaire, un genre féminin ?, op. cit.

RIPA Yannick, 1999, Les femmes, actrices de l'Histoire. France, 1789-1945, Paris, SEDES.

SCHMITT Jean-Claude, 1978, « Histoire des marginaux », in LE GOFF et al. (dir.), La Nouvelle Histoire, Paris, Retz, pp. 344-369.

SCHWARTZ Olivier, 1990, Le monde privé des ouvriers. Hommes et femmes du Nord, Paris, PUF. 
SCHWARZER Alice, 1975, La petite différence et ses grandes conséquences, Paris, Editions des femmes.

SCOTT Joan Wallach, 1986, « Gender : a useful category of historical analysis », American Historical Review, vol. 91, 5.

SILVER Marie-France et GIROU SWIDERSKI Marie-Laure, 2000, Femmes en toutes lettres. Les épistolières du XVIII siècle, Oxford, Voltaire Foundation.

THÉBAUD Françoise, 1986, Quand nos grand-mères donnaient la vie. La maternité en France dans l'entre-deux-guerres (version abrégée de la thèse de troisième cycle), Presses universitaires de Lyon.

-, 1998, Ecrire l'histoire des femmes, Paris, ENS Éditions.

VEAUVY Christiane et PISANO Laura (dir.), 1997, Paroles oubliées. Les femmes et la construction de l'État-nation en France et en Italie (1789-1960), traduction de Parole inascoltare, (1994), Paris, A.Colin.

VERDIER Yvonne, 1979, Façons de dire, façons de faire. La laveuse, la couturière, la cuisinière, Paris, Gallimard.

\section{NOTES}

1. Au Centre de Recherches Historiques (EHESS). Ce groupe fonctionne depuis 1978.

2. Duby et Perrot 1991.

3. Parmi les livres qui ont dressé un bilan historiographique, il faut citer : Thébaud 1998 ; Ripa 1999 ; Bard 2001 ; Godineau 2003.

4. Magloire 2002.

5. Profession de foi.

6. Voir Jean Baubérot, « De la femme protestante », in Duby et Perrot 1991 (t. 4) : 206-207.

7. Les tentatives répétées de modification de la loi sur l'avortement le montrent. Le dernier avatar étant l'amendement voté par l'Assemblée Nationale le 27 novembre 2003 créant un délit d'interruption involontaire de grossesse qui semble donner au fœtus une existence juridique propre, indépendante en quelque sorte de la mère.

8. Foucault $1984: 16$.

9. Entre autres, Schwarzer 1975.

10. Alain Corbin (1984) note ainsi que le seul mot de « plaisir » demeure banni de la table des matières de la thèse universitaire.

11. Schwartz 1990.

12. Dans un récent ouvrage, Ruwen Ogier (2003:110) reprend cet argument. En revanche, dit-il « aucun Juif ou Noir n'irait jusqu'à dire que le racisme ou l'antisémitisme sont bons pour les Noirs ou Juifs » Le racisme regroupant tous les actes contre un représentant d'une race, on en déduit que la pornographie regroupe tous les actes contre une femme. Il donne aussi comme référence Wendy Mc Ellroy 1995. Patrick Baudry (2001) affirme qu'entre 20 et 30\% de la clientèle est féminine dans les achats d'objets de sex-shop.

13. Ogivie 2002.

14. On rappellera l'émission de Mireille Dumas sur la prostitution, étonnante dans son propos général qui établissait une opposition entre prostituées françaises et étrangères, entre celles qui choisissaient et celles qui subissaient, et qui se concluait sur la revendication d'une française de bouter hors nos frontières les étrangères ! 
15. Botte 2003. Voir aussi l'affaire des femmes nigérianes obligées de se prostituer pour dettes et terrorisées par l'instrumentalisation du rituel vaudou.

16. Voir Dufourmantelle 2003 ; Marzano 2003 ; Cités 2003 ; Cosmopolitiques 2003.

17. Haase-Dubosc et Viennot 1991.

18. Dans leurs enquêtes sur Minot dans le Châtillonnais, entreprises en 1966.

19. C. Dauphin et al. 1986.

20. Cosandey 2000.

21. Entre autres, Farge 1991.

22. Godineau 1988.

23. Dauphin et al. 1997 et 2001.

24. Mathieu 1985.

25. Bourdieu 1998.

26. Bourdieu 1998.

27. Schwartz 1990.

28. Frader et Rose 1996 ; Davidoff 1995 ; Downs 2002.

29. Godineau $2003: 4-5$.

30. Entre autres Auslander et Zancarini-Fournel 1995.

31. Perrot 1998.

32. Thébaud 1998.

33. Duby et Perrot $1991:$ t.1, introduction.

34. Verdier 1979.

35. « Dires de femmes » est le titre d'une partie de L'Invention du quotidien de Michel de Certeau 1980 : vol. 2.

36. Pénélope 1979 : 2.

37. Ibidem.

38. Duby et Perrot 1991, vol.1, «Écrire l'histoire des femmes » : 9.

39. Schmitt 1978.

40. En 1833. Cité par Bulciolu $1980: 27$.

41. Régnier 1998.

42. Ainsi, aux Paroles d'hommes (Badinter 1989), s'ajoutent les Opinions de femmes (Fraisse 1989).

43. C'est ce que souligne le titre de l'ouvrage de Veauvy et Pisano 1997, traduit en français par Paroles oubliées.

44. Marini 1991 : 275-296.

45. Planté $1989: 15$.

46. De Piaggi 1993. Je m'appuie sur cet ouvrage pour résumer cette problématique.

47. Figures les plus en vue : Hélène Cixous, Annie Leclerc, Catherine Clément, Luce Irigaray, Julia Kristeva.

48. Yvonne Verdier, Françoise Zonabend, Martine Ségalen, Agnès Fine.

49. Dauphin et al. 1986.

50. Clio $16 / 2002: 145-166$.

51. Didier 1981 ; Planté 1989. ; van Dijk 2001 ; Silver et Girou Swiderski 2000 ; Hesse 2001. Cette liste n'est pas exhaustive.

52. Planté 1998.

53. Fabre $1993 ; 1997$.

54. Albert 1993 : 37-94 ; Lahire 1997 : 145-161 ; Fine 2000 ; Clio, 16/2002.

55. Golberg Moses et Wahl Rabine 1993.

56. Héritier 1996 ; 2002. 
57. Cf. le titre significatif de Hélène Cixous et Annie Leclerc: La venue à l'écriture, 1977.

58. En particulier travaux de Knibiehler et Fouquet 1980 ; Thébaud 1986.

59. Iacub 2002 ; Badinter 2003.

\section{RÉSUMÉS}

Militante et réflexive, l'histoire des femmes a aussi besoin d'exprimer ses doutes et ses inconforts. Rattrapée par l'actualité, la violence et nombre d'événements cruels et tragiques, par des incertitudes majeures vécues par l'ensemble du monde intellectuel, elle pose aujourd'hui en cet article collectif des interrogations et des inquiétudes. Ce travail à plusieurs (les participantes du Groupe d'histoire des femmes du $\mathrm{CRH}$ ) cherche à mettre à plat ce qui actuellement se dérobe à notre connaissance, en partant de notions-clés souvent utilisées comme consentement, sexualité, écriture, patriarcat, etc. Chacune de ces notions est discutée à la lumière des brouillages actuels, des temporalités nouvelles, de l'avènement triomphant du corps marchandise, de la pauvreté grandissante, etc.

Women's history, a militant and self-reflexive endeavor, needs to express its doubts and its difficulties. The contemporary world, with its violence, its cruel and tragic events, raises questions for all intellectuals and academics, affected by the uncertainties of the present, and all the more so for women historians and historians of women and gender. This collective article (written by the participants of the Women's History Group of the Centre de Recherches Historiques - CNRS/EHESS) addresses these issues by returning to key concepts that have been utilized over the last few decades - such as consent, sexuality, patriarchy, etc. Each term is analyzed in the light of current debates and new temporalities, from recent discussions about the body (and its commercialization) to growing inequalities, etc.

\section{INDEX}

Mots-clés : classes sociales, consentement, écriture, féminisme, paroles, patriarcat, histoire des femmes, sexualité, politique

\section{AUTEURS}

\section{YANNICK RIPA}

Groupe d'histoire des femmes du Centre de Recherches Historiques 